\title{
El vínculo con el hogar familiar en el segundo decenio del siglo XXI. Posibles diferencias de apego entre jóvenes creyentes y no creyentes
}

\section{Javier Aznar Sala'}

Resumen: El desigual proceso religioso que se está dando entre la misma juventud española en el siglo XXI, permite que establezcamos unas pautas para comprender mejor posibles diferencias entre jóvenes creyentes y no creyentes en un tema tan sensible como la emancipación del hogar. La juventud es cada vez menos sensible a cuestiones trascendentes, pero todavía quedan franjas juveniles que afirman sentirse vinculadas al hecho religioso. Nuestro trabajo busca, por medio de la entrevista de estas bases poblaciones, hallar posibles diferencias entre unos y otros. La juventud habita un mismo escenario público y son hijos de una misma generación, por lo que encontrar distinciones entre ellos puede resultar interesante para la ciencia social.

Palabras clave: Vínculo; juventud; emancipación; religión; valores; trabajo; análisis comparativo.

Fecha de recepción: I5 de abril de 202I.

Fecha de admisión definitiva: I de diciembre de 2021 .

'Este artículo se encuentra dentro de la línea de investigación Vínculos interpersonales y sus implicaciones en la familia de la Universidad Católica de Valencia "San Vicente Mártir" (Facultad de Filosofía, Letras y Humanidades). 
The bond with the family home in the second decade of the 21 st century. Possible differences of bonding between young non-believers and young religious

\begin{abstract}
The unequal religious process that is taking place among the Spanish youth itself in the 21 st century, allows us to establish guidelines to better understand possible differences between young believers and non-believers on such a sensitive issue as the emancipation of the home. The youth is less and less sensitive to transcendental issues, but there are still youth stripes that claim to feel linked to the religious fact. Our work seeks, through the interview of these population bases, to find possible differences between them. Youth live in the same public arena and are children of the same generation, so finding distinctions between them can be interesting for social science.
\end{abstract}

Key words: Bonding; youth; emancipation; religion; values; job; comparative analysis.
L'attachement au foyer familial dans la deuxième décennie du XXle siècle. Différences possibles dans l'attachemententre les jeunes croyants et les non-croyants

Résumé: Le processus religieux inégal qui a lieu parmi les jeunes espagnols au XXle siècle nous permet d'établir quelques lignes directrices pour mieux comprendre les différences possibles entre les jeunes croyants et non-croyants sur une question aussi sensible que l'émancipation du foyer. Les jeunes sont de moins en moins sensibles aux questions transcendantales, mais il y a encore des jeunes qui prétendent se sentir liés à la religion. Notre travail vise, en interrogeant ces bases de population, à trouver les différences éventuelles entre l'une et l'autre. Les jeunes vivent le même scénario public et sont les enfants d'une même génération. Trouver des distinctions entre eux peut donc être intéressant pour les sciences sociales.

Mots clé: lien; jeunesse; émancipation; religion; valeurs; travail; analyse comparative.

\section{Introducción}

Cada vez son más los estudios que hablan de las enormes dificultades que tiene la juventud para poder emanciparse del hogar familiar. A las distintas crisis económicas y la precariedad laboral se unen otros factores como la dilatada vida estudiantil y especialización académica en forma de idiomas, prácticas y estancias en el extranjero. Los padres son los que sostienen en muchos casos a una generación poco dada a proyectos de futuro y obligada a vivir el instante con pocas posibilidades de asumir el reto de la independencia del hogar. La posible pérdida de una serie valores entre la juventud actual influyen decisivamente en sus posibles elecciones. La generación de sus padres y abuelos está marcada por valores religiosos y la suya es indiferente a estos. El presente estudio procura comprender mejor la realidad de jóvenes entre los 18 y los 24 años y, además, establecer una comparativa entre aquellos que son indiferentes a lo religioso y otros que dicen ser creyentes, para 
intentar vislumbrar si es verdad que los valores religiosos inciden en las posibles elecciones a la hora de emanciparse más prematuramente del hogar familiar como ocurría con sus padres y abuelos.

Una reciente encuesta del CIS, elaborada en abril del 2020 y que se presenta como estudio $n^{\circ} 3279$ (CIS, abril 2020), sitúa las creencias de la población española en torno al $61,2 \%$ en referencia al catolicismo. En cambio, la indiferencia, el agnosticismo y el ateísmo se mueven en torno al $36,1 \%$ de la población. Es verdad que las bases juveniles se inclinan más hacia la segunda vertiente y, especialmente, entre el sector masculino más que entre el femenino. No obstante, podemos visualizar en la figura que sigue una acentuada polarización poblacional entre los que se consideran creyentes y los que no. La sociedad española es plural en sus manifestaciones pero no lo es tanto en el plano de las creencias, pues todavía predomina un claro sentimiento de pertenencia a la Iglesia Católica frente a otras posibles manifestaciones religiosas que suponen el 1,8\% del total.

\section{FIGURA I. Índices de religiosidad entre la juventud española}

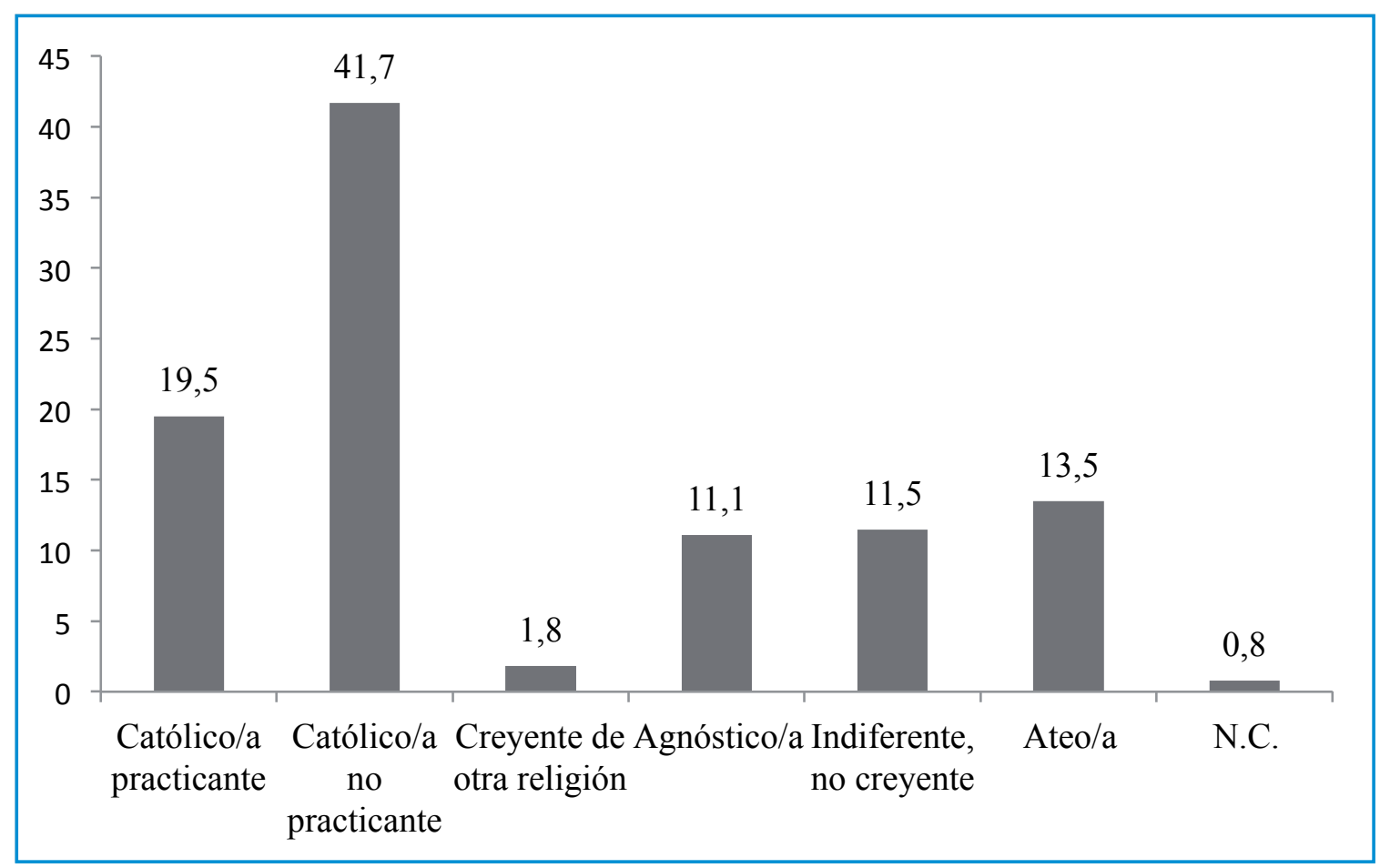

Fuente: Encuesta del CIS Abril de 2020 sobre pertenencia religiosa de la población española. Estudio $n^{\circ} 3279$, pregunta 35 . 


\section{Metodología}

El concepto de juventud entra de lleno en el marco del actual trabajo y para ello es necesario esclarecer el rango considerado de edad. En el ámbito europeo se suele utilizar el intervalo de juventud que va desde los 16 a los 24 años. El grupo de edad que consideraremos en este estudio se adecua a estos parámetros, pero más centrado en el baremo que va de los 18 a los 24 años y que engloba la categoría de estudiantes universitarios. Un desafío para el objetivo de esta investigación versa sobre la elección de los posibles candidatos universitarios que proceden de diversas partes de España y que han coincidido en la Universitat de València: Sevilla, Córdoba, Vigo, Valladolid, Extremadura, Valencia, Alicante y Castellón.

Sabemos que la muestra no es lo representativa que hubiéramos deseado y, por tanto, aplicable a todo el grueso de la población juvenil, pero sí que nos permite comprender algunas de las pautas y valores para poder establecer comparaciones posteriores. Las respuestas y posibles elecciones que nos han trasladado en las encuestas nos aportan una información que resulta útil para encuadrar nuestro análisis y contrastar la literatura escogida para centrar la investigación.

Los métodos utilizados en este trabajo se sustentan sobre la revisión literaria y la investigación empírica basada en la entrevista, realizando un total de treinta entrevistas y apoyándonos en una adecuada revisión científica. El propósito de nuestro estudio consiste en reunir diferentes perspectivas sobre la juventud en referencia a su jerarquía de valores y si existe alguna relación entre tales opciones y la posible emancipación del hogar de sus padres, según se sea o no sensible al hecho religioso. La muestra final se sostiene sobre un grupo de 15 jóvenes que estudian un grado sanitario de entre 18 y 24 años y otro sector de 15 jóvenes con creencias religiosas más heterogéneo en sus estudios dentro del mismo recinto académico.

Además, siguiendo al pensador canadiense Charles Taylor, se subraya que la juventud actual está más polarizada que nunca lo haya estado entre aquellos que dicen no tener ninguna convicción religiosa y los que sí afirman tenerlas. La polarización se refiere a las notables diferencias que se dan entre ambos estratos poblacionales y que se acentúan cada vez más. No nos interesa en este trabajo establecer porcentajes de creyentes y no creyentes, como hemos hecho al inicio a modo de encuadre, sino de entender cuáles son las posibles diferencias entre ambos perfiles respecto al vínculo que se establece con el hogar de referencia paterno o materno.

Para llevar a cabo el trabajo se han diseñado una serie de entrevistas semiestructuradas con preguntas abiertas y la finalidad de ayudar al joven a expresar mejor su opinión sobre cada cuestión planteada en la encuesta. El listado de preguntas 
ha sido utilizado de forma flexible buscando las cuestiones más relacionadas con el perfil y la experiencia de los informantes. Se han llevado a cabo entre febrero de 2019 y marzo de 2020. Una vez recibidas y estudiadas las encuestas se ha realizado un posterior análisis cuantitativo y cualitativo sobre el contenido de los resultados recibidos. Del total de 30 jóvenes elegidos para la encuesta han respondido 26 , lo que supone un $86 \%$ del conjunto. Las preguntas las hemos analizado con el mismo orden con que fueron diseñadas y con los posibles valores finales que se podían elegir en el orden de sus preferencias.

\section{FIGURA 2. Encuesta repartida entre los jóvenes}

a) ¿Cómo percibes la posibilidad futura de emancipación del hogar familiar de los jóvenes de tu generación?; ¿qué condiciones deberían darse para que un joven pudiera tener su propia autonomía?; ¿ Crees que es algo que tu generación desea o se encuentra cómoda cómo está?

b) Enumera en la tabla de más a menos importante los siguientes valores en tu baremo de prioridades:

- Familia, amor de pareja, tener un buen trabajo, viajar, salud, emancipación, espiritualidad, amistades, tener familia propia e hijos.

Fuente: Elaboración propia.

Las conclusiones de la encuesta se irán desplegando a lo largo del presente estudio, por lo que no se trata de desglosar los datos y separarlos del resto, sino de enmarcar sus resultados a lo largo de la exposición. Los datos extraídos nos permitirán vislumbrar las posibles pautas por las que transitan los jóvenes en edad universitaria y las posibles tendencias y diferencias que se dan. Por lo tanto buscamos dos variables muy claras: 1) Las posibles diferencias entre jóvenes en vistas a la emancipación del hogar; 2) Cuál es la jerarquía de valores elegida. La recogida de los datos se ha puntuado de forma numérica en orden a sus prioridades, asignando 9 puntos al valor elegido en primer lugar y 1 punto al último. Una vez sumado el conjunto en una tabla genérica donde aparecen todos y cada uno de los valores con sus puntuaciones; el valor más votado adquiere la fracción máxima de 100 sobre 100, el segundo más elegido 89 sobre 100, el tercero 78 sobre 100, el cuarto 67 sobre 100, el quinto 56 sobre 100, el sexto 45 sobre 100, el séptimo 34 sobre 100, el octavo 23 sobre 100 y el noveno 11 sobre 100. Todos ellos se han divido en fracciones de 11 para establecer una graduación que nos permita visualizar en una tabla y en una figura la escala de valores votados de mayor a menor. 


\section{Hogares y nidos. La emancipación en la juventud}

El hogar familiar se ha ido convirtiendo en los últimos años en una especie de campamento base para aquellos jóvenes que ya han superado la adolescencia. Decimos esto en el sentido siguiente: lo normal para el joven en el hogar familiar siempre ha sido la transitoriedad; es decir, pasado un tiempo los jóvenes tenían el compromiso tácito de salir del hogar para empezar su propio proyecto de vida a modo de ley no escrita. La situación ha variado notablemente en lo que se refiere a la emancipación de los jóvenes del hogar de sus padres, pues muchos de ellos se sienten cómodos yendo y viniendo de sus hogares respectivos como un lugar de seguridad desde el cual realizar cuantas actividades se les presenten. Un joven postmoderno puede pasar temporadas en su casa y, al mismo tiempo, pasar largas estancias fuera de ella, pero con la idea de vuelta. Esta situación permite no ligarse a ninguna hipoteca ni asumir cualquier proyecto personal que implique un "para siempre". Los jóvenes son conocidos como la generación del boomerang kids, que se refiere a una generación con estudios pero atrapados entre dos crisis: la de la burbuja inmobiliaria del 2008 y la actual del 2020 que será conocida como de la COVID-19. Recientes estudios de la Fundación BBVA confirman esta tesis que venimos señalando al indicar que el $65^{\prime} 1 \%$ de los jóvenes, de entre 16 y 34 años, siguen viviendo en el hogar familiar. Además indican que esta realidad ha ido creciendo en un porcentaje superior al del $7 \%$ en los últimos diez años:

El porcentaje de jóvenes de entre 16 y 34 años que no está emancipado ha aumentado del 58,1\% en 2008 al 65,1\% diez años después. En la decisión de abandonar el hogar familiar influyen factores relacionados con el mercado de trabajo, el nivel de formación alcanzado o las condiciones de accesibilidad a la vivienda, además de factores culturales. La tasa de emancipación de los jóvenes con un contrato indefinido asciende al $62 \%$, frente al $36,8 \%$ de los que disponen de un contrato temporal. La continuación de los estudios también puede retrasar la salida del hogar familiar, ya que únicamente el $8 \%$ de los jóvenes que cursa formación reglada está emancipado. Por último, ante las dificultades de acceso a la vivienda, en 2017 casi el $60 \%$ de los hogares sustentados por jóvenes opta por una vivienda en alquiler o cedida por familiares, mientras que en 2008 el 60,5\% se decantaba por una vivienda en propiedad (BBVA, 2019).

El grado de emancipación del hogar se va ralentizando progresivamente y únicamente a partir de la treintena años se empieza a plantear la posibilidad real de emanciparse y que se ha retrasado 10 años con respeto a la generación de sus padres. Un joven necesita de una adecuada seguridad en el plano laboral para dar el salto hacia una vida independiente, además son muchos los que prolongan 
sus estudios y viajes casi de forma indefinida, como es el caso de másteres de especialización o de prolongadas estancias en el extranjero para aprender idiomas o acumular experiencias.

En la universidad de Heilsenberg (Alemania), se acaba de realizar un interesante estudio sobre la incidencia que tiene la presencia de los jóvenes en el hogar de los padres. Las conclusiones de este estudio llevado a cabo en toda Europa con una muestra de más de 55.000 personas, resulta esclarecedor e ilustrativo. Los padres de aquellos hijos que no alcanzan a cobrar la independencia necesaria para emanciparse del hogar presentan un cuadro mayor de ansiedad, depresión o falta de autoestima. En cambio, aquellos padres que comprueban que sus hijos poseen la capacidad de afrontar su vida con ciertas garantías y tomar el timón de la misma en forma de independencia, seguridad económica, laboral y afecti$\mathrm{va}$, suelen presentar mayores cuotas de bienestar personal (Becker, Kirchmaier \& Trautmann, 2019).

No podemos olvidar que hace apenas algunos años se hablaba de la generación ni-ni (jóvenes que ni estudian ni trabajan) como de un auténtico problema social y generacional. Tanto es así que la Unión Europea realizó un estudio por medio de la Agencia Europea de Estadística en el año 2013, donde concluía que más de 15 millones de jóvenes europeos de entre 20 y 34 años presentaban un perfil ni-ni. Uno de cada seis europeos queda clasificado en esta franja de población inactiva o improductiva (Eurostat, 2019). En cambio, la tendencia a partir del 2018 se revierte hacia un tipo de jóvenes denominados como la generación sí-sí, con una actitud mucho más positiva hacia la formación, el estudio y el trabajo. Los datos son muy llamativos, pues la diferencia entre varones y hembras -en su condición de ni-ni- ahonda hacia una brecha significativa entre sexos. Mientras que el porcentaje de chicos que no tenían acceso al trabajo ni al estudio era del $12,2 \%$ el de las chicas representa un $20,9 \%$. Es más, las diferencias se acrecientan en países del Este de Europa y, especialmente llamativo es el caso de la República Checa, donde los varones suponen el $4,6 \%$ y el de las féminas se sitúan en un alarmante 25,9\% (Eurostat, 2019).

Lo que es evidente es que el mercado laboral para los jóvenes está cambiando a pasos agigantados y estamos pasando de la generación ni-ni a la generación sí-sí. No obstante, el mercado laboral está profundamente dividido entre jóvenes muy formados $y$, otros, poco cualificados y con bajas posibilidades de insertarse en el mercado laboral. Las desigualdades quedan patentes al comprender que muchas de las chicas jóvenes que son calificadas dentro del grupo ni-ni sí que trabajan en el hogar, pero esta labor no es reconocida ni remunerada como 
trabajo doméstico (Pérez Adán, 2020). Como se extrae de otra investigación de la Fundación Europea para la Mejora de las Condiciones de Vida y de Trabajo (Eurofound), se concluye que es urgente reintegrar cuanto antes a este perfil de mujeres jóvenes que, después de tener un hijo, son tasadas dentro del grupo ni-ni para que pasen a ser consideradas dentro del sector de mujeres trabajadoras (Espacio Público, 2019). La verdad es que se dedican al cuidado de jóvenes y de mayores y su labor ha de ser reconocida socialmente, no hacerlo redunda en una inadmisible injusticia social.

Pocos son los trabajos en el presente que no requieren de cierta especialización y, por tanto, de un mímica formación académica. Tanto es así que algunos sociólogos indican que "las universidades son productoras de grupos profesionales sobre los que pivota la estructura ocupacional de las sociedades modernas" (Giner, 2010, p. 325). Los jóvenes que no han tenido la posibilidad de especializarse académicamente tienen acceso a trabajos ocasionales y volátiles, donde lo que prima es la inseguridad laboral y la dificultad real de construir un proyecto personal.

\section{FIGURA 3. Escala de valores de los actuales jóvenes según su perfil creyente $o$ indiferente}

\begin{tabular}{|c|c|c|c|c|c|}
\hline \multicolumn{6}{|c|}{ Valores elegidos de mayor a menor } \\
\hline \multirow{9}{*}{ 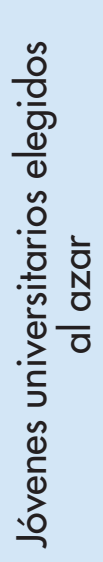 } & Familia & 100 & \multirow{9}{*}{ 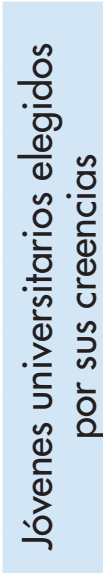 } & Espiritualidad & 100 \\
\hline & Salud & 89 & & Familia & 89 \\
\hline & Amistad & 78 & & Amistad & 78 \\
\hline & Buen trabajo futuro & 67 & & Salud & 67 \\
\hline & Pareja & 56 & & Familia e hijos propios & 56 \\
\hline & Emanciparse & 45 & & Pareja & 45 \\
\hline & Viajar & 34 & & Emanciparse & 34 \\
\hline & Familia e hijos propios & 23 & & Buen trabajo futuro & 23 \\
\hline & Espiritualidad & 11 & & Viajar & 11 \\
\hline
\end{tabular}

Algunos autores coinciden en el hecho de que la edad de la juventud actual se resiste a pasar y entrar en la vida adulta: "la juventud pasó de ser un estado transitorio biológico entre la adolescencia y la adultez a un valor en sí mismo que había que eternizar de todas formas" (Piscitelli, 2020, p. 309). Este porcentaje es tan alto en la actualidad que algunos pensadores contemporáneos han llegado a 
hablar de los «adultescentes» (Fabri, 2009). En la tabla que sigue hemos elegido una serie de valores para que fueran ordenados por los jóvenes actuales de entre 18 a 24 años en una valoración que va de más a menos importante para que podamos comprender mejor su jerarquía de valores y acercarnos a su realidad social más inmediata.

\section{Comparativa entre perfiles juvenil sobre la emancipación}

Una vez organizados por su orden de elección, según han sido valorados los distintos ítems de la tabla, se pueden deducir algunas cuestiones que nos acercan a la comprensión de los jóvenes, con valores religiosos o sin ellos, merced a los datos de la encuesta. La tabla nos permite comprobar que se dan ligeras variaciones en las elecciones según se tengan o no algún tipo de valores trascendentes. Algo llamativo es el poco valor que se le otorga a la espiritualidad por parte del primer grupo de jóvenes no religiosos, en cambio, contrasta con el alto valor que le conceden aquellos que sí se consideran creyentes, tanto es así que eligen la espiritualidad en primer lugar y como fundamento de sus posteriores elecciones. Se trata de una primera apreciación que resulta interesante y que parece confirmar la polarización social a la que aluden diversos estudios de los que nos hacemos eco.

Para hablar de la población juvenil que asume para sí valores religiosos utilizamos el término religamiento que ha sido ampliamente estudiado:

Este religamiento supone una búsqueda de trascendencia por parte de nuevas bases poblacionales cuando lo único que se esperaba era la desafección religiosa en la postmodernidad. [...] secularización y religamiento se están dando al mismo tiempo y en un mismo espacio (Anónimo, 2020).

La figura que sigue a continuación permite visualizar las semejanzas y las diferencias entre ambos perfiles de jóvenes según sean creyentes o indiferentes. 


\section{FIGURA 4. Escala de valores de los actuales jóvenes según su perfil creyente $o$ indiferente}

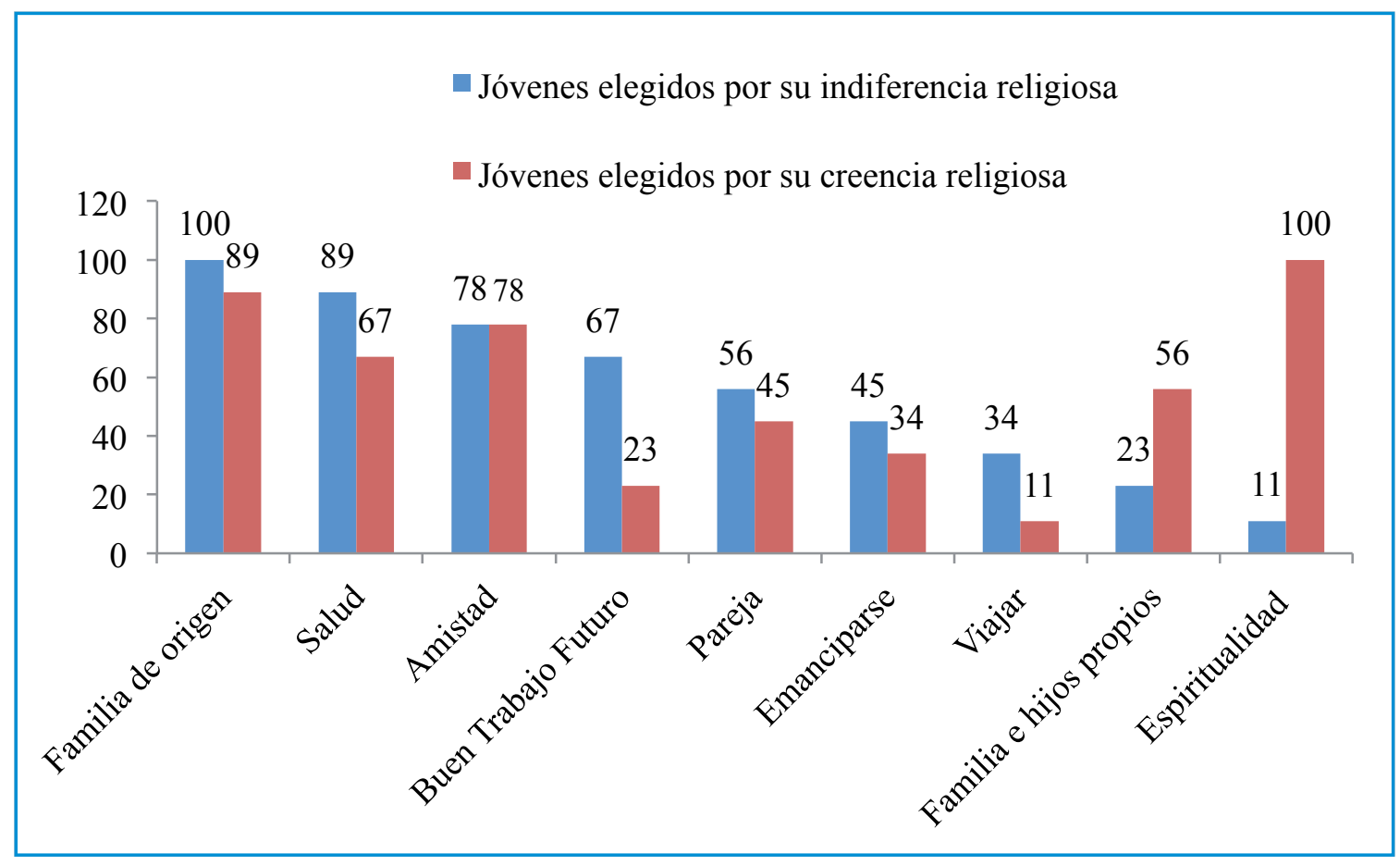

Fuente: Elaboración propia a partir de los datos recogidos en la encuesta.

La elección de un valor trascendente en último o primer lugar configura el resto de elecciones y las jerarquiza de distinta manera. Los jóvenes siguen valorando en igual medida la institución familiar a la que pertenecen y poseen un gran concepto de ella. No tienen como prioridad emanciparse como lo desearon sus padres, más bien se encuentran cómodos en el hogar familiar, aunque esta apreciación acepta algún matiz que es necesario subrayar.

La emancipación -por parte de los jóvenes no religiosos- es escogida en un sexto lugar y se valoran antes otro tipo de elecciones. En relación a este dato concurre la baja valoración que se le otorga a formar una familia propia. Es como si esta franja de jóvenes poseyeran otros intereses relacionados con el disfrute inmediato: salud, amistad, buen trabajo y pareja. Destaca el alto valor de la amistad que se da entre todos los jóvenes actuales, como uno de los atributos con los que la juventud se identifica mayoritariamente. Se trata de jóvenes que valoran altamente la amistad que suele estar unida a la diversión y que ocupa numerosas horas tanto físicas como en las redes sociales. 
Entre los jóvenes no religiosos la emancipación del hogar paterno se pospone hasta bien entrada la treintena de años. Es evidente que postergar esta realidad incide notablemente en el tipo de familia y número de hijos que se puedan tener en el futuro. Como nos indica la tabla de valores "formar familia propia y tener hijos" es la penúltima elección entre estos jóvenes pero no lo es entre jóvenes creyentes, que perciben la pareja en función de un tránsito hacia el proyecto familiar con hijos. El pensador canadiense Charles Taylor considera el actual escenario como marcado por una cultura materialista que "nos asienta firmemente en un tiempo secular cada vez más racionalizado y medido" (Taylor, 2006, p. 214).

Los jóvenes en su conjunto sí que suelen ser sensibles a causas que suponen una lucha global o posicionamiento frente a lo que como sector social consideran que representan injusticias sociales como la lucha por el planeta, la igualdad o los derechos de la mujer. En este sentido sí que parecen presentar bastante homogeneidad y pocas diferencias entre unos y otros. Los jóvenes conviven en un mismo imaginario social que difiere del de sus padres. Mientras que sus padres tenían claras referencias a valores centrados en el bien de la colectividad, lo que Charles Taylor denomina una sociedad (porous self); es decir, abierta a valores trascendentes como el proyecto de vida, la espiritualidad, el cuidado de los hijos, etc.; la juventud actual vive un imaginario diverso o sociedad menos abierta y referenciada en uno mismo (buffered self), centrados en su bienestar personal sin una tendencia clara a construir un proyecto común (Taylor, 2007).

Nuestros informantes señalan que la emancipación no es algo que esté entre sus prioridades y ha de darse después de numerosos requisitos o seguridades que lo permitan. Todos coinciden, creyentes e indiferentes, en la dificultad de tal cometido, aunque el sector de los jóvenes creyentes presenta ciertos matices que invitan a pensar que tienen mayor conciencia del sacrificio y la responsabilidad que supone salir del hogar. De todas las opiniones cualitativas hemos escogido algunas que muestran lo que venimos señalando. La variable numérica se refiere a los jóvenes escogidos por su indiferencia religiosa y la alfabética la de aquellos con perfiles creyentes. 


\section{FIGURA 5. Valoración de la emancipación según diversas perspectivas de jóvenes creyentes o no}

\begin{tabular}{|c|}
\hline Jóvenes elegidos al azar \\
\hline $\begin{array}{l}\text { Joven 1: "Yo pienso que mi generación } \\
\text { no exige ningún tipo de emancipación. } \\
\text { En algunos casos sería prácticamente } \\
\text { imposible, me refiero a la gente que } \\
\text { quiera continuar los estudios". }\end{array}$ \\
\hline
\end{tabular}

Joven 2: "Creo que la generación de hoy en día se encuentra bastante cómoda a diferencia de años atrás que solían independizarse a edad más temprana".

Joven 6: "Pienso que mi generación tiene menos anisas de libertad que las anteriores y nos sentimos cómodos en casa de los padres".

Joven 9: "Pienso que muchos adolescentes se encuentran cómodos en sus casas ya que no se tienen que preocupar por los gastos que suelen tener los adultos".

\section{Jóvenes elegidos por sus valores religiosos}

Joven B: "Pienso que uno se independiza sin tener un respaldo económico que asegure una estabilidad. Esta mentalidad choca con la que tenemos inculcada (trabajar, ser indefinido, comprar una casa, independizarse...)".

Joven C: "Si antes de la pandemia las posibilidades eran difíciles, cuánto más ahora con los recortes de personal y las mermas en los salarios. La autonomía se da antes de salir de casa".

Joven D: "Lo que se necesitan son ayudas o acceso a trabajos que permitan independizarse. Creo que la mayoría sí lo desea".

Joven E: "Para poder adquirir autonomía el joven debería comprender que la vida también conlleva sacrificios en pos del bien común y de la convivencia. Creo que mi generación no quiere este cambio, somos realmente egoístas".

Fuente: Elaboración propia a partir de la encuesta.

El sector de jóvenes creyentes parece estar más abierto a la emancipación pero admite que la comodidad del hogar por un lado y la falta de recursos para construir una vida propia por otro, puede mucho más que el proyecto propio. La salida del hogar se produce, pero de forma eventual y en pisos del alquiler con otros jóvenes o con largas estancias en el extranjero. 


\section{FIGURA 6. Valoración de la emancipación según diversas perspectivas de jóvenes creyentes o no}

\section{Jóvenes elegidos al azar}

Joven 3: "Yo veo que es posible obviamente, pero a veces pensamos que es fácil llevar una casa adelante cuando no es así para nada, y menos todavía si estudiamos al mismo tiempo.

Joven 10: "Las condiciones son que tengas un puesto de trabajo, donde se cobre bien y no una miseria, para así poder pagarte la casa o el piso".

Joven 12: "Veo que es una posibilidad con mucha responsabilidad, las condiciones para que un joven pudiera tener su propia autonomía, serían tener trabajoy un sueldo propio, responsabilidades propias. Creo que en mi generación pocos serán los que se independicen".

\section{Jóvenes elegidos por sus valores religiosos}

Joven F: "Los jóvenes sólo podríamos independizarnos accediendo a compartir un piso con al menos otra persona más. Se vive para trabajar, en lugar de trabajar para vivir".

Joven G: "Existe una dependencia paterna en muchos aspectos que en ocasiones es debida a la falta de madurez del individuo o a la educación recibida".

Joven H: "Poseemos un deseo muy grande de autonomía total y yo deseo tener mi vida y mi hogar y proyecto personal. Pero no todos están dispuestos a hacerse cargo de sí mismos, cuando tienen ya a sus padres que les proveen con lo necesario".

Fuente: Elaboración propia a partir de la encuesta.

Existen estudios que indican que las fluctuaciones económicas inciden decisivamente en la posibilidad de plantearse la salida del hogar. La variabilidad de la salida del hogar según momentos económicos de expansión o de recesión tan acentuados como los actuales son decisorios para la juventud. Una economía sometida a constantes fluctuaciones es demasiada incertidumbre para unos jóvenes que desean volar del nido y los convierte a su pesar en la generación boomerang kids:

Este fenómeno es particularmente susceptible a los ciclos económicos. Durante la fase reciente de expansión económica el porcentaje de españoles entre 18 y 34 años que dejaron de vivir con los padres pasó del 35,1\% en 2002 al $44,9 \%$ en 2007. Sin embargo, desde principios de 2008 la crisis económica ha empujado proporciones crecientes de jóvenes emancipados a volver a casa de sus padres. 
En este mismo periodo la tendencia a emanciparse se ha ralentizado: en el cuarto trimestre de 2004 el número de los que se independizaron incrementó en un 5,6\% respecto al año anterior, a finales de 2008 este aumento fue tan sólo del 1,5\% (CJE, 2009) (Gentile, 2010, p. 181).

El esfuerzo que tendría que realizar un joven para adquirir su primera vivienda está alrededor del $85 \%$ de su sueldo. Es cierto que el alquiler de una vivienda parece más factible y menos gravoso, a la par que supone menos riesgos, pero de igual modo "el pago de una renta libre, con fianzas y avales, excedería del $56 \%$ de su salario" (Gentile, 2010, p. 185). Los jóvenes han quedado en medio de un vacío que se presenta en recurrentes de crisis económicas y que ralentiza sus aspiraciones:

Quien vive lejos de su casa (leaving away from home) por razones de estudio o de trabajo está más expuesto a trayectorias de vuelta al hogar cuando haya acabado sus experiencias. Por otra parte, no todos los que dejan su familia con la idea de no volver atrás (leaving home) pueden definirse realmente independientes, ya que sus padres pueden sostener su emancipación desde un punto de vista financiero, como en el caso de comprar una vivienda, o práctico, a la hora de acogerles en casa con frecuencia y de cubrir sus tareas domésticas con regularidad (Gentile, 2010, p. 170).

Los jóvenes del presente parecen estar obligados a permanecer en el hogar sin tener expectativas reales de salida en muchos casos. Los ciclos naturales se subvierten dadas las condiciones socioeconómicas que les ha tocado vivir. Además de ser jóvenes post-millenials se caracterizan por ser mileuristas en el caso de acceder a un contrato laboral. Se trata de trabajos eventuales que no garantizan la total independencia en un contexto cada vez más incierto y fluctuante. En terminología sociológica se habla de jóvenes con «trayectorias fallidas» que impide su normal desarrollo (Walther \& Stauber, 2002). Así pues, se genera en ellos la sensación de cierto desengaño y desilusión por el establishment.

los jóvenes, agobiados por tanta negatividad radio-televisada, asumen cierto escepticismo respecto al espacio público. [...] un leve nihilismo de fondo lo impregna todo, y los jóvenes intentan neutralizar sus efectos desmoralizantes con mayores dosis de diversión (Cerezo \& Gómez, 2006, p. 124).

Ante la actual situación hay algunos autores, como el filósofo surcoreano ByungChul Han (2020), que perciben el mundo del trabajo como carente de esperanza y en sintonía con nuestro análisis. Se deriva de su estudio una división de la historia en base al trabajo: primero servil y después convertido en una especie de trabajo explotador o auto-explotador en la modernidad. Dejamos, por tanto, para el futuro un tipo de trabajo mucho más creativo y humanizado donde prevalezca el espíritu de servicio y una posibilidad digna de emancipación. 


\section{Conclusiones}

La posibilidad real de emancipación entre la población juvenil es una de las cuestiones más debatidas en el presente, pues va unida a la posibilidad de la independencia del hogar familiar y aparejada un trabajo seguro y con buenas prestaciones económicas. Paradóiicamente, las constantes y fluctuantes crisis económicas que venimos arrastrando desde 2008 suponen un techo de cristal para una juventud más preparada que nunca y con menores posibilidades reales de establecer un proyecto personal en comparación con las generaciones precedentes. Las dificultades son muchas y en forma de pocos recursos y seguridades.

Nuestra sociedad actual se caracteriza por dar poco valor a cuestiones trascendentes y en tal hecho algunos sociólogos han querido ver la causa de la ralentización de la salida del hogar entre los jóvenes actuales. De alguna manera las generaciones precedentes, con personas más o menos religiosas a título particular, venían acompasadas de un imaginario social referido a valores religiosos y que impulsaba a la independencia del hogar familiar y al matrimonio con hijos. En tal sentido hemos querido realizar una comparación en el presente entre jóvenes que no dicen poseer ninguna creencia religiosa con otros jóvenes que sí dicen tenerlas. El objetivo era claro, comprobar si unos y otros poseen algún tipo de diferencias en lo que se refiere a la salida del hogar y asumir como propio el proyecto personal de independencia en referencia a un hogar propio y unos posibles hijos futuros.

La realidad de nuestro estudio comparativo ha resultado bastante esclarecedora, pues se trata de jóvenes de entre 18 a 24 años de distintos perfiles académicos y religiosos. Entre ambos sectores, no importa el porcentaje de los mismos, sino su posicionamiento ante la realidad estudiada, no se dan diferencias notables en la visión que tienen sobre la salida del hogar. En ambos casos la percepción de dificultad ante la posibilidad de emancipación es muy acusada debido a la falta real de recursos y de seguridades. Es por ello que esta posibilidad de salida se ha ido retrasando en el tiempo y ya se abre una brecha de diez años con respecto de la generación de sus padres (en tan solo una generación). Todo ello hace que la juventud sea un periodo de se alarga hasta pasada la treintena de años y con una inseguridad notable respecto a pensar en un hogar y familia propia.

Señalar que hemos encontrado algunas pequeñas diferencias en los resultados de las entrevistas y sobre todo en la elección de valores. Los jóvenes poco creyentes se centran en valores más inmediatos y materiales como el viajar, el amor de pareja como proyecto definitivo, el tener muchas y buenas amistades o poseer un buen trabajo futuro. En cambio, los jóvenes creyentes sitúan en el primer lugar 
de elección un valor tan poco valorado por los otros como la espiritualidad. De alguna manera esta elección trascendente tamiza el resto de valores escogidos y establece una jerarquía más inclinada al amor de pareja pero percibido como algo transitorio y no como proyecto definitivo, en vistas a tener un hogar y una familia propia e hijos.

Los jóvenes creyentes manifiestan que no es tan importante tener una gran seguridad para construir su propio proyecto vital en forma de hogar y de familia $y$, en cambio, los no creyentes parecen pedir un mayor rango de seguridades para dar el paso decisivo. Ambos grupos valoran enormemente la amistad y sobre todo la institución familiar, cosa que les iguala como generación pero difieren sensiblemente en sus metas. Nos parece un estudio interesante porque refleja la realidad social actual y que nos permite comprender mejor lo que viven los jóvenes. Se trata de una generación más polarizada que nunca entre aquellos que no creen en Dios y los que dicen creer, no tanto en número sino en su posicionamiento ante la vida.

\section{Bibliografía}

AzNAR, F. J. (2016). Religamiento y juventud en un contexto de secularización: jóvenes valencianos en el segundo decenio del siglo XXI, Universidad Católica de Valencia, España.

- (2020) "María y el religamiento", en Pérez AdÁN, J., (ed.), Sociología mariana, Pamplona, Eunsa.

BBVA (2019). El 65,1\% de los jóvenes españoles de entre 16 y 34 años sigue viviendo en el hogar familiar, un porcentaje 7 puntos superior al de hace diez años. (Consulta 04/06/2020), de BBVA. Sitio web: https://www.fbbva.es/ noticias/651-los-jovenes-espanoles-16-34-anos-sigue-viviendo-hogar-familiarporcentaje-7-puntos-superior-al-diez-anos/

Becker, C., Kirchmaier, I., y Trautmann, S. T. (2019). "Marriage, parenthood and social network: Subjective well-being and mental health in old ag", PloS one, $14(7)$.

BERGER, P. L. y LUCKMANN, T. (1995). La construcción social de la realidad, Madrid, Amorrortu.

Bourdieu, P. (2003). Cuestiones de sociología, Madrid, Istmo.

Caballero, F. (1986). "Jóvenes sin vivienda", Alfoz, 27: 16-17. 
Centro InVESTIGACIONES SOCIOlÓGICAs (abril 2020). Barómetro Especial de Abril 2020, Estudio n ${ }^{\circ}$ 3279. (Consulta 29/07/2020), de CIS. Sitio web: http://www.cis.es/cis/ export/sites/default/-Archivos/Marginales/3260_3279/3279/es3279mar.pdf

Cerezo, J. J. y Gómez, P. J. (2006). Jóvenes e Iglesia, Madrid: SM.

DREHER, R. (2018). La opción benedictina, Madrid, Encuentro.

EsPACIO PÚBLICO (2019). Brecha entre sexos de jóvenes latinoamericanos. (Consulta 17/04/20) de Espacio Público, de Espacio Público. Sitio web: https://www. espaciopublico.cl/

EUROSTAT (2019). 1 in 6 young people not in employment or education. (Consulta 29/05/20). Sitio web: https://ec.europa.eu/eurostat/web/products-eurostatnews/-/DDN-20190627-1 ?inheritRedirect=true\&redirect=\%2Feurostat\%2Fhome $\% 3 \mathrm{~F}$

FABBRI, E. (2009). Adolescencia: promesa de un mundo mejor, Madrid, Paraninfo.

GeNtile, A. (2010). "De vuelta al nido en tiempos de crisis. Los boomerang kids españoles", Revista de estudios de juventud, 90(10), 181-203.

GINER, S. (2010). Sociología, Barcelona, Península.

HAN, Byung-Chul (2020). La desaparición de los rituales, Barcelona, Herder.

MACINTYRE, A. (2015). Tras la virtud, Barcelona, Critica.

MIRET, P. (2005). "Pautas territoriales en la emancipación juvenil en España, cohortes de nacimiento 1924-1968", Papeles de Geografía, 41-42: 161-176.

Pérez AdÁn, J. (Ed.) (2018). Sociología de la experiencia religiosa, Pamplona, Eunsa.

- (Ed.) (2019). Sociología mariana, Pamplona, Eunsa.

Piscitelu, A. (2020), "La sociedad de consumo", en Pérez Adán (ed.), Economía y salud social, Pamplona, Eunsa, 309-335.

TAYLOR, Ch. (2006). Imaginarios sociales modernos, Barcelona, Paidós.

- (2007). A secular age, Cambirdge, Harvard University Press.

Walther, A. y Stauber, B. (2002) Misleading Trajectories. Integration Policies for Young Adults in Europe?, Opladen, Leske and Budrich. 